\title{
Investigation of the Microstructure of a Sintered Sample Obtained from Powder Steel X13
}

\author{
S.V. Khardikov ${ }^{1, *}$, E.V. Ageeva ${ }^{1}$, V.I. Kolmykov ${ }^{1}$, and V.I. Serebrovsky ${ }^{2}$ \\ ${ }^{1}$ Southwest State University, 305040, Kursk, Russian Federation. \\ ${ }^{2}$ Kursk State Agricultural Academy, 305021, Kursk, Russian Federation.
}

\begin{abstract}
This article presents the results of a study of the microstructure of a sintered sample made of alloy X13 electroerosive materials obtained in butyl alcohol. It has been established that during the sintering of electroerosive materials made of alloy X13 by the method of spark plasma sintering, the main elements on the surface of the particles are $\mathrm{Fe}, \mathrm{Cr}, \mathrm{Mn}$, and the particles have a generally regular spherical shape.
\end{abstract}

\section{Introduction}

Among the intensively developing areas of modern research, a special place is given to small-sized objects, for example, powders of various metals. The unique microstructure of the powders gives them a number of new properties compared to conventional materials. Nowadays, powders are of great interest.

Powder metallurgy is a branch of technology that includes the manufacture of powders from metals and their alloys and the production of blanks and products from them without melting the main component. Powder metallurgy methods can be used to create materials from various components with sharply differing properties and melting points, new materials with a variety of physical and mechanical properties. Powder metallurgy is used both for the creation of fundamentally new materials and products from them and for the manufacture of the widest range of general-purpose structural parts.

The widespread use of X13 steel in various industries leads to a large accumulation of its waste that requires processing. Currently, there are many ways to dispose of the metal waste for reuse. However, the disadvantages of the known methods are increased energy consumption, multi-stage process [1-7].

The most promising method for processing metal waste is the method of electroerosive dispersion (EED), which is distinguished by the environmental friendliness of the process and relatively low energy consumption.

Comprehensive theoretical and experimental studies are needed to develop technologies for the practical use of powder materials obtained from X13 alloy waste and to assess the efficiency of their use. The work aimed to study the microstructure of sintered samples from alloy X13 electroerosive materials.

\footnotetext{
*Corresponding author: hardikov1990@mail.ru
} 


\section{Materials and Methods}

The study of the microstructure was carried out on an OLYMPUS GX51 inverted optical microscope, which is designed to obtain in reflected light: bright-field and dark-field images; differential interference contrast (DIC) imaging; images in polarized light. Maximum magnification of the microscope: x1000 (interchangeable objectives x5, x10, $\mathrm{x} 20, \mathrm{x} 50, \mathrm{x} 100)$. The OLYMPUS GX51 microscope is additionally equipped with a precision scanning automated table PS 11, a digital microscope video camera SIMAGIS 2P-2C and an automated image analysis system "SIAMS Photolab" (SIMAGIS Research) and ImageScope M. The image analysis system "SIAMS Photolab" is a software product designed for processing and analysis of images obtained using digital and analog photo and video cameras, as well as scanners during micro and macro photography. Image processing in "SIAMS Photolab" is performed in a chain of interconnected cells containing the original image, the results of intermediate processing stages, the final processed image, and measurement results in the form of numbers, graphs, and histograms. After creating a chain for processing a new sample according to the specified algorithm, it is enough to replace the original image. At the same time, the user can visually control and manually adjust the parameters of any stage of processing. In addition to automated processing, the system allows editing images in manual and semi-automatic modes.

By the method of optical microscopy, a study of the microstructure of the samples (on a transverse section) was carried out. The surface of the samples was ground and polished. Grinding was carried out with metallographic paper with coarse (No. 60-70) and fine grain (No. 220-240). During grinding, the sample was periodically turned $90^{\circ}$. The abrasive particles were washed off with water and subjected to polishing on a wheel with suspensions of metal oxides ( $\mathrm{Fe} 3 \mathrm{O} 4, \mathrm{Cr} 2 \mathrm{O} 3, \mathrm{Al} 2 \mathrm{O} 3$ ). After reaching a mirror finish, the surface of the section was washed with water, alcohol and dried with filter paper [8-14].

The study of the microstructure was carried out on a Rigaku Ultima IV X-ray diffractometer. Applications: phase analysis of samples, quantitative phase analysis of samples, determination of areas of coherent scattering and microstresses, texture analysis. Features of the Ultima IV series diffractometer: goniometer radius $185 \mathrm{~mm}$ at the output beam, variable width slits. Allows you to keep the irradiated sample surface unchanged. $\Theta$ / vertical type goniometer for all three configurations, adapted to accommodate a wide range of optional optical components. A new model of the high-speed X-ray detector D / teX Ultra, which allows 100 times faster measurements than the previous detectors of this company. It is a detector with high count rate, high energy resolution and low noise. Multi purpose attachment MPA-IV $\chi$ (kai) - $\varphi$ (phi) - Z stage. Sample changer (10 cuvettes). Software: qualitative and quantitative phase analysis, ICDD PDF-2 diffractogram database, crystallinity analysis, residual stress analysis, construction of forward and reverse pole figures, orientation distribution function. Specifications. Radiation source: small-sized using a high-frequency converter, maximum power - $3 \mathrm{~kW}$, tube voltage $-20-60 \mathrm{kV}$, tube current - 2-60 mA, tube anode material - Cu, focus size - 0.4 x $12 \mathrm{~mm}$. Goniometer: $\Theta$ / vertical type, specimen motionless. Scanning method - independent scanning of each axis $\Theta s$ or $\Theta \mathrm{d}$; scanning mode with connected axes $\Theta \mathrm{s} / \Theta \mathrm{d}$. Goniometer radius - 185mm; range of scanning angles in the mode of coupled axes $\Theta \mathrm{s} / \Theta \mathrm{d}$ from -30 to $+1620(2 \Theta)$; $\Theta \mathrm{s}$ axes from -1.50 to +810 , $\Theta d$ axes from -950 to +1200 ; scanning step for the $\Theta s$ or $\Theta d$ axis $0.0001-60$; in coupled axes mode $0.0002-120(2 \Theta)$. Scanning speed in coupled axes mode $\Theta \mathrm{s} / \Theta \mathrm{d} 0.020 \sim 1000(2 \Theta)$, independently of each axis $0.010 \sim 500$; positioning speed $5000 / \min (2 \Theta)$. Slits: with a controllable width for the output and diffracted beams. Two standard sets of Soller slits for focusing and pseudo-parallel beam geometry. Adjustment: fully automatic for goniometer, amplitude discriminator, counter, optical units and additional attachments. Detector: Scintillation counter with a linearity of $700000 \mathrm{imp}$. 
(standard), single-axis semiconductor detector D / teX Ultra with a sensitivity that is two orders of magnitude higher than that of a scintillation counter [15-20].

\section{Results}

The results of the study of the sample are shown in Figure 1.

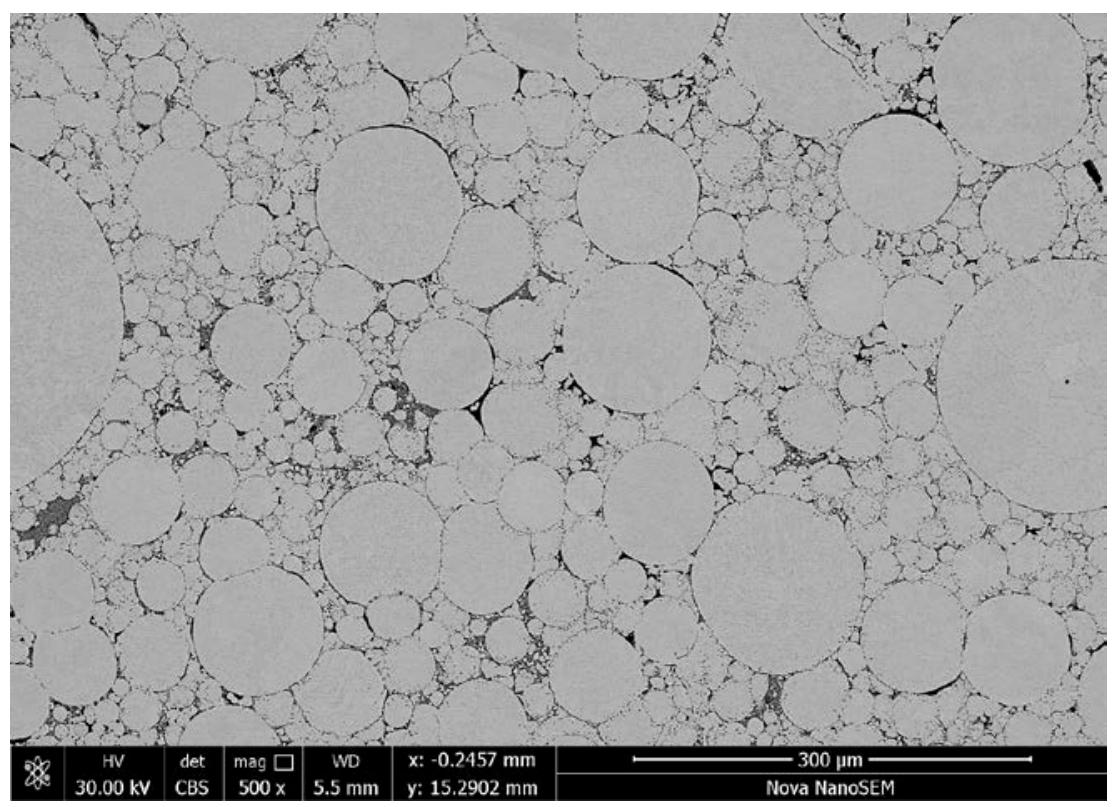

Fig. 1. The microstructure of the sintered sample

The picture shows that the sintered sample has a fine-grained microstructure, which significantly increases its operational properties.

The results of studying the elemental composition of the surface of sample X13 are presented in Table 1 and Figure 2.

Table 1. Elemental composition of the surface of sample X13

\begin{tabular}{|c|c|c|}
\hline Element & $\mathrm{Wt}, \%$ & $\mathrm{At}, \%$ \\
\hline $\mathrm{O}$ & 1.00 & 3.35 \\
\hline $\mathrm{Co}$ & 2.03 & 1.85 \\
\hline $\mathrm{Si}$ & 0.78 & 1.49 \\
\hline $\mathrm{Cr}$ & 12.91 & 13.29 \\
\hline $\mathrm{Mn}$ & 12.78 & 12.45 \\
\hline $\mathrm{Fe}$ & 70.39 & 67.47 \\
\hline $\mathrm{Ni}$ & 0.11 & 0.10 \\
\hline Total & 100.00 & 100.00 \\
\hline
\end{tabular}




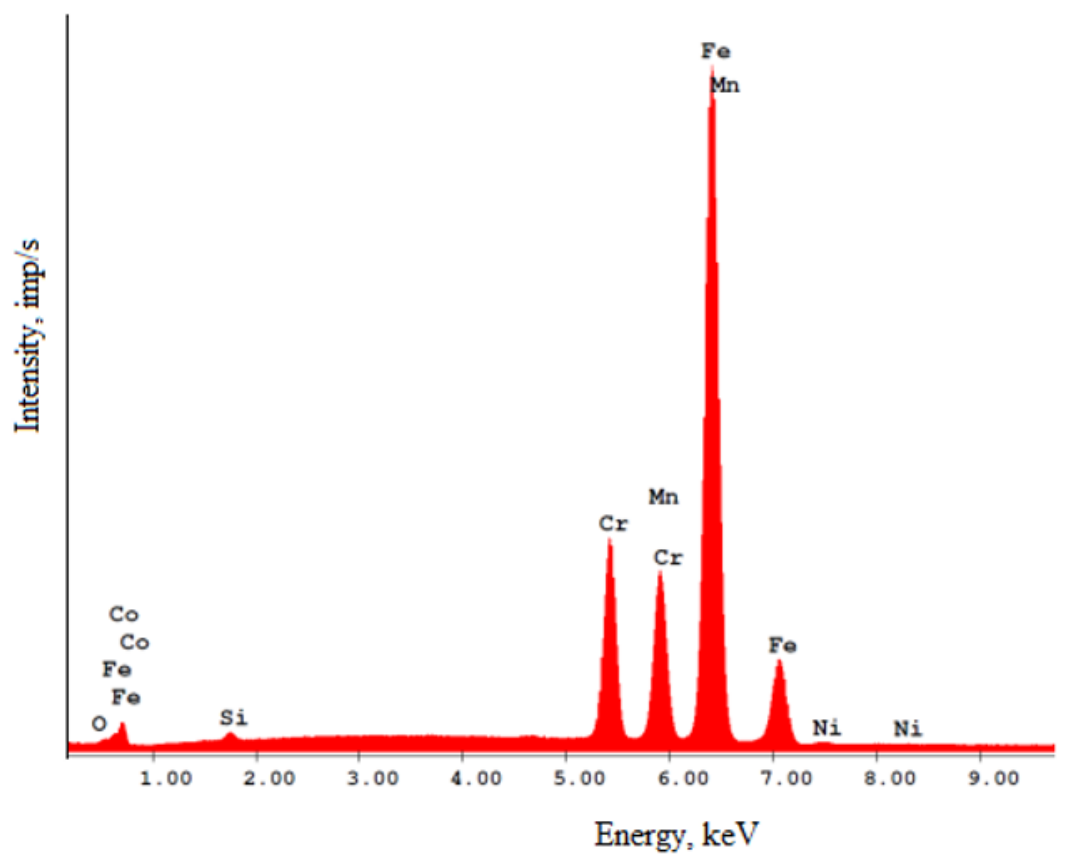

Fig. 2. X-ray of a sintered sample

It is seen that the main elements on the surface of the sintered sample are $\mathrm{Fe}, \mathrm{Cr}, \mathrm{Mn}$.

\section{Conclusion}

In the course of the study, it was found that the sintered sample has a fine-grained microstructure, which significantly increases its operational properties, the main elements on the surface of the sintered sample are Fe, Cr, Mn. It can be concluded that the resulting sintered products have high-performance characteristics and can be used in mechanical engineering.

The work was supported by a scholarship of the President of the Russian Federation for young scientists and graduate students (SP-945.2019.1).

\section{References}

1. A.A. Lipatov, RER, 33, 3, 144-149 (2013)

2. A.M. Adaskin, A.A. Vereshchaka, A.S. Vereshchaka, J. of Frict. and W., 34, 208-213 (2013)

3. V.L. Bibik, MSF, 762, 777-781 (2013)

4. E.V. Azarova, E.A. Levashov, V.G. Ralchenko, A.P. Bolshakov, E.E. Ashkinazi, Metall., 54, 523-529 (2010)

5. Z. Qiao, X. Ma, W. Zhao, H. Tang, B. Zhao, J. of All. and Comp., 462, 416-420 (2008)

6. K. Maruyama, T. Nonaka, H.Y. Kim, Intermet., 13, 1116-1121 (2005) 
7. E.V. Ageev Patent 2449859, Russian Federation, C2, B22F9 / 14. Installation for producing nanodispersed powders from conductive materials, applicant and patent holder Southwestern State University. - No. 2010104316/02; application 02/08/2010; publ. 05/10/2012, 4.

8. E.V. Ageev, E.V. Ageeva, Bull. of mech.eng., 11, 51-57 (2013)

9. E.V. Ageev, B. A. Semenikhin, R. A. Latypov, Fund. Prikl. Probl. Tekhn. Tekhn., 5, 39-42 (2010)

10. T.N. Oskolkova, E.A. Budovskikh, Metal. Sci. Heat Treat, 55, 96-99 (2013)

11. J. Karlsson, A. Snis, H. Engqvist, J. Lausmaa, J. of Mat. Proc. Techn., 213, 2109-2118 (2013)

12. D.D. Gu, W. Meiners, K. Wissenbach, R. Poprawe, IMR, 57, 133-164 (2012)

13. N. Radek, Maint. and Rel., 4, 10-16 (2009)

14. A.V. Ribalko, O. Sahin, Surf. \& Coat.Techn., 168, 129-135 (2003)

15. Z. Chen, Y. Zhou, Surf. \& Coat.Techn, 201, 1503-1510 (2006)

16. I.V. Galinov, R.B. Luban, Surf. \& Coat.Techn, 79, 9-18 (1996)

17. E.V. Azarova, E.A. Levashov, V.G. Ralchenko, Transl. from Metall. 8, 50-55 (2010)

18. A. Pereverzev., E. Ageev., MATEC Web of Conf., 298, 00037 (2019)

19. E.V. Ageev, S.V. Khardikov, E.A. Vorobyev, A.A. Sysoev, MATEC Web of Conf., 298, 00127 (2019)

20. R.A. Latypov, E.V. Ageeva, G.R. Latypova, MATEC Web of Conf., 298, 00125 (2019) 